\title{
Photodissociation spectroscopy of $\mathrm{ClCN}$ in the vacuum ultraviolet region
}

\author{
Kazuhiro Kanda ${ }^{a, *}$, Mitsuhiko Kono ${ }^{\text {b,1 }}$, Takashi Nagata ${ }^{b, 2}$, Atsunari Hiraya ${ }^{b, 3}$, \\ Kiyohiko Tabayashi $^{\text {b, }}{ }^{\text {, Kosuke Shobatake }}{ }^{\text {b, } 5}$ \\ ${ }^{a}$ Laboratory of Advanced Science and Technology for Industry, Himeji Institute of Technology, Kamigori, Hyogo 678-1205, Japan \\ ${ }^{\mathrm{b}}$ Institute for Molecular Science, Okazaki, Aichi 444-8585, Japan
}

Received 21 February 2000

\begin{abstract}
The quantitative photofragment fluorescence spectroscopy, using the synchrotron radiation as an exciting light source, was applied to study the Rydberg and high-lying valence states of $\mathrm{ClCN}$ observed as congested structures in the vacuum ultraviolet region. The absolute cross-section and quantum yield for the $\mathrm{CN}\left(\mathrm{B}^{2} \Sigma^{+}-\mathrm{X}^{2} \Sigma^{+}\right)$emission produced in the photodissociation process of $\mathrm{ClCN}$ were determined in the wavelength range 105-145 nm $\left(69000-95200 \mathrm{~cm}^{-1}\right)$. The quantum yield for the $\mathrm{CN}\left(\mathrm{B}^{2} \Sigma^{+}\right)$production takes a maximum value of $\approx 0.13$ at $\approx 84000 \mathrm{~cm}^{-1}$. The emission of $\mathrm{CN}\left(\mathrm{B}^{2} \Sigma^{+}-\mathrm{X}^{2} \Sigma^{+}\right)$transition was found to be partially polarized with respect to the direction of the electric vector of the excitation synchrotron radiation. The polarization anisotropy of this emission, which depends on the symmetry of absorption transitions into the photodissociative states of $\mathrm{ClCN}$ was measured as a function of exciting wavelength. The relative cross-section for the production of $\mathrm{CN}\left(\mathrm{A}^{2} \Pi_{i}-\mathrm{X}^{2} \Sigma^{+}\right)$emission was also determined. Based on the measured photochemical properties of the high-energy electronic states, the observed bands of the Rydberg and intravalence transitions are assigned. (c) 2000 Elsevier Science B.V. All rights reserved.
\end{abstract}

\footnotetext{
* Corresponding author.

${ }^{1}$ Present address: Solar-Terrestrial Environment Laboratory, Nagoya University, Toyokawa, Aichi 442-8507, Japan

2 Present address: Department of Basic Science, Graduate School of Arts and Sciences, Meguro-ku, Tokyo 153-8902, Japan.

${ }^{3}$ Present address: Department of Material Science, Faculty of Science, Hiroshima University, Higashi-Hiroshima, Hiroshima 739-8526, Japan.

${ }^{4}$ Present address: Department of Chemistry, Graduate School of Science, Hiroshima University, Higashi-Hiroshima, Hiroshima 739-8526, Japan.

${ }^{5}$ Present address: Department of Materials Chemistry, School of Engineering, Nagoya University, Chikusa-ku, Nagoya 464-8603, Japan.
}

\section{Introduction}

In the vacuum UV (VUV) photodissociation process of $\mathrm{ClCN}$, the electronically excited radicals of $\mathrm{CN}\left(\mathrm{B}^{2} \Sigma^{+}\right)$and $\mathrm{CN}\left(\mathrm{A}^{2} \Pi_{i}\right)$ are produced as the photofragments and the subsequent $\mathrm{CN}\left(\mathrm{B}^{2} \Sigma^{+}-\right.$ $\left.\mathrm{X}^{2} \Sigma^{+}\right)$and $\mathrm{CN}\left(\mathrm{A}^{2} \Pi_{i}-\mathrm{X}^{2} \Sigma^{+}\right)$emissions have been observed in the UV and visible region and in the near infrared region, respectively. The knowledge of the high-lying electronic states is important to discuss the photodissociation dynamics of $\mathrm{ClCN}$ in the VUV region. However, the congested features of the VUV absorption spectrum of $\mathrm{ClCN}$ prevented us from the fully understanding the property of the precursor states, $(\mathrm{ClCN})^{*}$, through 
which fragmentation proceeds. Although electronic excited states of $\mathrm{ClCN}$ have been investigated by several workers, their assignments are not all consistent and the peaks in the $\lambda<120 \mathrm{~nm}$ have not been assigned [1-7].

Through systematic work, we have investigated the highly excited states of the cyanogen halide leading to the production of $\mathrm{CN}(\mathrm{A})$ and $\mathrm{CN}(\mathrm{B})$ radicals. In our previous works, the transition peaks observed in the VUV absorption spectra of $\mathrm{BrCN}$ and ICN have been assigned to the Rydberg series and the intravalence transitions [8,9]. The same transitions are expected to appear in the photoabsorption spectrum of $\mathrm{ClCN}$, because $\mathrm{ClCN}$ has the chemical properties similar to these molecules. On the other hand, the spectral positions must be shifted because the ionization potentials of $\mathrm{ClCN}$ are higher than those of $\mathrm{BrCN}$ and ICN.

In the present study, the absolute cross-section and quantum yield for the production of $\mathrm{CN}(\mathrm{B})$ in the VUV photodissociation process of $\mathrm{ClCN}$ were determined as a function of excitation wavelength ranging from 105 to $145 \mathrm{~nm}$. In addition to these quantitative measurements, the polarization anisotropy of the resulting $\mathrm{CN}\left(\mathrm{B}^{2} \Sigma^{+}-\mathrm{X}^{2} \Sigma^{+}\right)$emission was measured by taking advantage of the linear polarization of the synchrotron radiation. The polarization anisotropy of the fragment emission with respect to the direction of the electric vector of incident radiation gave us an information on the symmetry of the initial electronic transition into the dissociative state. Measurements for the polarization anisotropy of $\mathrm{CN}(\mathrm{B}-\mathrm{X})$ observed in the VUV photodissociation process of $\mathrm{ClCN}$ have been reported by Simons and coworkers at several excitation wavelengths in the $126-154 \mathrm{~nm}$ region [3,4] and by Zare and coworkers at $157.6 \mathrm{~nm}$ [10]. In the present experiment, the polarization anisotropy of $\mathrm{CN}(\mathrm{B}-\mathrm{X})$ was measured as a function of wavelength ranging from 105 to $145 \mathrm{~nm}$ at intervals of $0.02 \mathrm{~nm}$. Our "close" measurement distinguished the tiny structures in the excitation function from the underlying diffuse bands and made the spectral assignments of the VUV absorption bands of ClCN reliable. The relative cross-section for the $\mathrm{CN}(\mathrm{A})$ production in the VUV photodissociation process of $\mathrm{ClCN}$ was also measured.

\section{Experimental}

The experimental apparatus and procedures employed in the present study have been described in the previous paper $[8,9,11]$. The measurement was performed at the BL2A stage of UVSOR in the Institute for Molecular Science. The synchrotron radiation provided by the $0.75-\mathrm{GeV}$ electron storage ring was dispersed by a 1-m Seya-Namioka monochromator (Hitachi SNM-2) and was introduced into the 12.3-cm-length reaction flow cell through the $\mathrm{LiF}$ window. Slit widths of the monochromator were $100 \mu \mathrm{m}$ for the measurement of the absorption and fluorescence excitation spectra, and $300 \mu \mathrm{m}$ for the measurement of the polarization anisotropy of $\mathrm{CN}(\mathrm{B}-\mathrm{X})$ emission. These resolutions of exciting light wavelength were about 0.1 and $0.3 \mathrm{~nm}$, respectively, which were estimated from the spectral width of the atomic absorption line of $\mathrm{Kr}$ at $123.6 \mathrm{~nm}$ and those of $\mathrm{Xe}$ at 119.20 and $146.96 \mathrm{~nm}$. A wavelength reading of the monochromator was calibrated against these atomic absorption lines. The uncertainty in the calibrated wavelength was estimated to be $\pm 0.03 \mathrm{~nm}$.

The $\mathrm{ClCN}$ sample was prepared by the reaction of $\mathrm{NaCN}$ with $\mathrm{Cl}_{2}$ in a $\mathrm{CCl}_{4}$ medium at $-10^{\circ} \mathrm{C}$ [12]. The pressure of sample in the reaction flow cell monitored with a capacitance manometer (Baratron Type 315) was typically 15-45 mTorr $(1$ Torr $=133.322 \mathrm{~Pa})$. The VUV photons passing through the cell were detected by a Hamamatsu R585 photomultiplier after conversion to visible fluorescence by sodium salicylate coated on the outside of the exit LiF window. The output signal from the photomultiplier tube was fed into a picoammeter (TDA AM-271A). The photoabsorption cross-section, $\sigma$, was determined from the ratio of the VUV photon fluxes through the flow cell measured with and without sample gas. The uncertainty of the absolute absorption crosssection was estimated to be $\approx 10 \%$ due to the uncertainty of the sample gas pressure.

The fragment emissions were collimated through a quartz lens attached on the side wall of the reaction flow cell and focused through another quartz lens to a detector. The $\mathrm{CN}(\mathrm{B}-\mathrm{X})$ emission in the UV and visible region was isolated by 
a band-pass filter (Toshiba C-39A) and detected by a photomultiplier (Hamamatsu R585). The emission of $\mathrm{CN}(\mathrm{A}-\mathrm{X})$ transition observed in the near infrared region was monitored with a combination of a sharp-cut filter (Toshiba R-65) and a photomultiplier (Hamamatsu R955) equipped with a photomultiplier cooler (Hamamatsu C659). The emission signal was acquired using an Ortec photon counting system. The emission cross-section determined in the present study corresponds to the cross-section for the production of $\mathrm{CN}(\mathrm{B}), \sigma_{\mathrm{B}}$, because the radiative decay can be regarded as the dominant deexcitation process for the $\mathrm{CN}$ formed in the $\mathrm{B}$ state. The absolute value of $\sigma_{\mathrm{B}}$ was scaled by a comparison of the intensity of the $\mathrm{CN}(\mathrm{B}-\mathrm{X})$ emission produced in the photodissociation of $\mathrm{HCN}$, for which the absolute emission crosssection has been reported [13]. The details about the scaling procedure to obtain the absolute emission cross-section from the emission intensity and relative optical responses of the detection systems were described in Ref. [9]. The relative uncertainty of $\sigma_{\mathrm{B}}$ was estimated to be $\approx 10 \%$. On the other hand, the absolute values of the crosssection for the production of $\mathrm{CN}(\mathrm{A}), \sigma_{\mathrm{A}}$, could not be determined, because the detection system for the near infrared region did not cover all the spectral region of $\mathrm{CN}(\mathrm{A}-\mathrm{X})$ transitions, as discussed in Ref. [9]. The quantum yield for the production of $\mathrm{CN}(\mathrm{B}), \Gamma_{\mathrm{B}}$, was calculated as the ratio of the emission cross-section to the absorption cross-section. The relative uncertainty of $\Gamma_{\mathrm{B}}$ was estimated to be $\approx 20 \%$.

The polarization anisotropy, $R$, is defined as

$R=\left(I_{\|} / I_{\perp}-1\right) /\left(I_{\|} / I_{\perp}+2\right)$,

where $I_{\|}$and $I_{\perp}$ are the intensities of the emission with the light component parallel and perpendicular to the direction of the electric vector of the incident radiation, respectively. In order to measure the polarization anisotropy of $\mathrm{CN}(\mathrm{B}-\mathrm{X})$ emission produced in the photodissociation process of $\mathrm{ClCN}$, two identical sets of a band-pass filter and a Glan-Taylor prism polarizer (KarlLambrecht MGTYA15) were mounted behind the collimating quartz lenses [8]. The absolute values of the $I_{\|} / I_{\perp}$ ratio for the $\mathrm{CN}(\mathrm{B}-\mathrm{X})$ emission were determined by using a photoelastic modulator (PEM, Hinds International Inc. PEM-80) and a multichannel scaler (MCS) at several excitation wavelengths, in order to eliminate the factors giving rise to the uneven signal levels of $I_{\|}$and $I_{\perp}$. The details in the PEM-MCS technique have been described in Ref. [11]. The statistical errors in the measurement of the $I_{\|} / I_{\perp}$ ratio gave rise to the uncertainty of $\sim 0.01$ of $R$.

\section{Results and discussion}

\subsection{Electronic structure of cyanogen chloride}

The molecular orbital (MO) configuration of $\mathrm{ClCN}$ in the ground state is given in the following [14-16]:

$$
(1 \sigma)^{2}(2 \sigma)^{2}(3 \sigma)^{2}(1 \pi)^{4}(4 \sigma)^{2}(2 \pi)^{4} ; \quad{ }^{1} \Sigma^{+}
$$

The ionization potentials, IP, the frequencies of the vibrational modes, $v_{1}, v_{2}$, and $v_{3}$ and spin-orbit splittings, SO, are listed in Table 1 . The three band systems are observed in the photoelectron spectrum of cyanogen chloride. The cyanogen chloride cations in these three lowest energy ionic states and the cyanogen chloride in the ground state have been reported to be linear [14-17].

The first band system (IP $=99770 \mathrm{~cm}^{-1}$ ) in the photoelectron spectrum are due to the removal of an electron from the $2 \pi \mathrm{MO}$, which originates from the combination of the $3 p$ orbital of the chlorine atom and the $\pi$ bonding orbital of the $\mathrm{CN}$ group out of phase. The splitting of this band system by spin-orbit coupling, i.e. the splitting between $\mathrm{ClCN}^{+}\left(\mathrm{X}^{2} \Pi_{3 / 2}\right)$ and $\mathrm{ClCN}^{+}\left(\mathrm{X}^{2} \Pi_{1 / 2}\right)$, is small in comparison with $\mathrm{BrCN}$ and ICN. The photoelectron intensity of the $\mathrm{X}^{2} \Pi_{3 / 2}$ component is much smaller than that of the $\mathrm{X}^{2} \Pi_{1 / 2}$ component. The second band system $\left(\mathrm{IP}=111300 \mathrm{~cm}^{-1}\right)$ is due to ionization of cyanogen chloride to the ${ }^{2} \Sigma^{+}$state, which corresponds to the removal of an electron from the $4 \sigma$ MO. The $4 \sigma$ orbital is regarded primarily as the lone pair localized on the nitrogen atom. The third band system (IP = $124000 \mathrm{~cm}^{-1}$ ) arises from the removal of an electron from the $1 \pi \mathrm{MO}$. This MO is the combination 
Table 1

Ionization potentials, vibrational frequencies and spin-orbit splittings $\left(\mathrm{cm}^{-1}\right)$

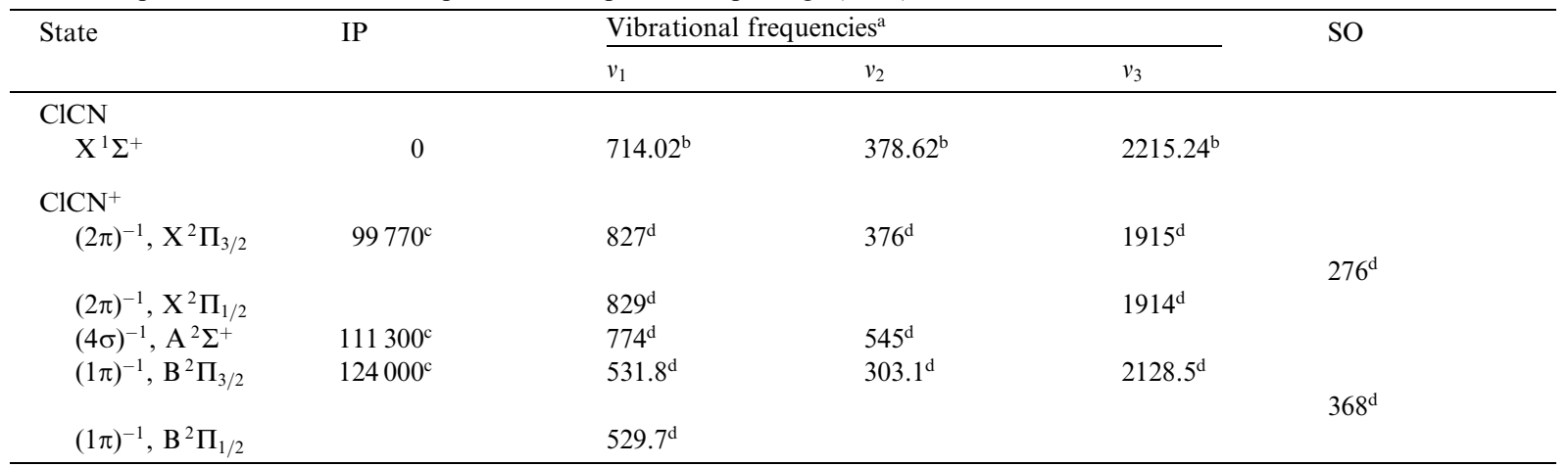

${ }^{a} v_{1}$ : C-N stretching, $v_{2}$ : bending, $v_{3}$ : $\mathrm{C}-\mathrm{Cl}$ stretching.

${ }^{\mathrm{b}}$ Ref. [17].

${ }^{\mathrm{c}}$ Ref. [14].

${ }^{\mathrm{d}}$ Ref. [20].

of the $3 p$ orbital of the chlorine atom and the $\pi$ bonding orbital of the $\mathrm{CN}$ group in phase.

\subsection{Absorption and excitation spectra}

Fig. 1 shows the VUV absorption spectrum of $\mathrm{ClCN}$ in the $69000-95200 \mathrm{~cm}^{-1}$ region. The spectral features and positions of the absorption bands observed in the present study are in good agreement with the photoabsorption spectrum of $\mathrm{ClCN}$, which has been previously reported by Simons et al. [3,4] and Felps et al. [5,7].

In Fig. 2(a), the absolute cross-section and quantum yield for the $\mathrm{CN}(\mathrm{B})$ production are

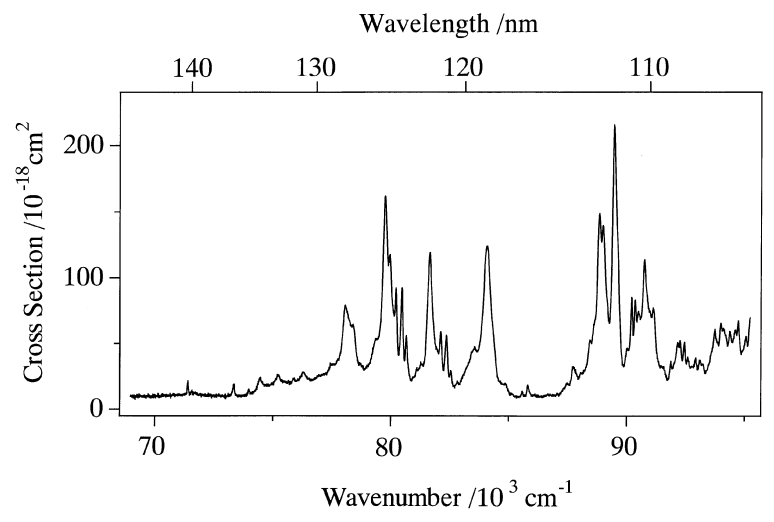

Fig. 1. Photoabsorption cross-section of $\mathrm{ClCN}$ in the $69000-$ $95200 \mathrm{~cm}^{-1}$ range. The spectral resolution is $\approx 0.1 \mathrm{~nm}$. plotted against the excitation wave number, $\tilde{v}$, in the range $69000-95200 \mathrm{~cm}^{-1}$. The excitation spectrum of $\mathrm{CN}(\mathrm{B}-\mathrm{X})$ emission formed from the VUV photodissociation of $\mathrm{ClCN}$ has been reported previously by Macpherson and Simons $[3,4]$ in the region $\tilde{v}<81900 \mathrm{~cm}^{-1}(122 \mathrm{~nm})$ while the absolute cross-section of the $\mathrm{CN}(\mathrm{B}-\mathrm{X})$ emission has not been determined in their studies. The dominant photodissociation channels in the $69000-95200 \mathrm{~cm}^{-1}$ region lead to the production of $\mathrm{CN}$ fragments in the $\mathrm{X}$ and/or A states, because the observed maximum value of $\Gamma_{\mathrm{B}}$ is only $\approx 0.13$ at $\approx 84000 \mathrm{~cm}^{-1}$.

Fig. 2(b) depicts the polarization anisotropy, $R$, of the subsequent $\mathrm{CN}(\mathrm{B}-\mathrm{X})$ emission as a function of the excitation wave number. The values of $R$ expected in the photodissociation of cyanogen halide have been discussed in Ref. [8]. By considering the fact that $\mathrm{ClCN}$ is a linear molecule and that the transition dipole moment responsible for the fragment $\mathrm{CN}\left(\mathrm{B}^{2} \Sigma^{+}-\mathrm{X}^{2} \Sigma^{+}\right)$emission is fixed along the molecular axis of the $\mathrm{CN}$ fragment, the limiting value of $R$ merely depends on the type of absorption transitions relevant to the photodissociation of $\mathrm{ClCN}$. In the case of parallel-type transition, when the transition dipole moment for the absorption of $\mathrm{ClCN}, \mu_{\mathrm{abs}}$, is parallel with the molecular axis, i.e., $\mu_{\text {abs }}$ is perpendicular to the angular momentum, $J$, theoretical limit of $R$ value is calculated to be 0.1 . On the other hand, when $\mu_{\mathrm{abs}}$ is perpendicular to 

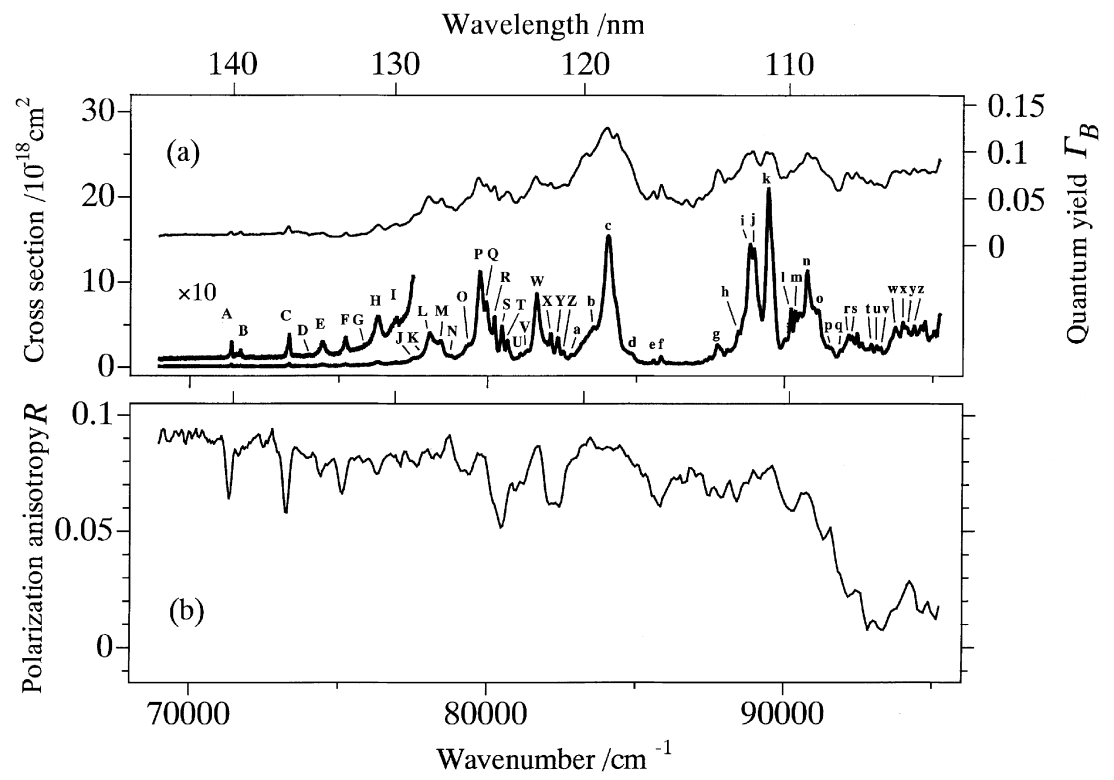

Fig. 2. (a) Absolute cross-section (-) and quantum yield (-) for the production of $\mathrm{CN}\left(\mathrm{B}^{2} \Sigma^{+}\right)$from $\mathrm{ClCN}$ in the $69000-94000 \mathrm{~cm}^{-1}$ range. (b) Polarization anisotropies of $\mathrm{CN}\left(\mathrm{B}^{2} \Sigma^{+}-\mathrm{X}^{2} \Sigma^{+}\right)$emission.

the molecular axis (perpendicular-type transition), i.e. $\mu_{\text {abs }} \| J$, the $R$ value will be 0.1 and -0.2 in the $\mathrm{P}$ and $\mathrm{R}$ branches and in the $\mathrm{Q}$ branch, respectively. In a predissociation process, the observed magnitude of $R$ is reduced to be less than these theoretical limit by the rotational motions of the dissociating $\left[\mathrm{ClCN}^{*}\right]$ molecule during its lifetime. Simons et al. have measured the $I_{\|} / I_{\perp}$ ratio of the $\mathrm{CN}(\mathrm{B}-\mathrm{X})$ emission from $\mathrm{ClCN}$ at several excitation wavelengths [3,4]. The $R$ values measured in the present study at the corresponding wavelengths agree well with those previously reported values $[3,4]$ within the experimental accuracy.

Fig. 3 shows the excitation spectrum and relative quantum yield for the $\mathrm{CN}\left(\mathrm{A}^{2} \Pi_{i}\right)$ production in the wave number region $69000-95200 \mathrm{~cm}^{-1}$. The wave number dependence of the cross-section for the $\mathrm{CN}(\mathrm{A})$ production resembles that for the $\mathrm{CN}(\mathrm{B})$ production in this region.

\subsection{Spectral assignments}

The photoabsorption peaks observed in the present measurement were interpreted in terms of excitations to the valence excited states and the
Rydberg states. The transition frequency, $\tilde{v}$, of a Rydberg series obeys the formula

$\tilde{v}=\mathrm{IP}-\frac{R_{\infty}}{(n-\delta)^{2}}$,

where $R_{\infty}$ represents the Rydberg constant (10973 $\mathrm{cm}^{-1}$ ) and $n$ is the principal quantum number. The quantum defect, $\delta$, is characteristic of a given Rydberg series, however, no regular Rydberg series are expected to appear in the photoabsorption spectra of cyanogen halide due to strong interaction between intravalence and Rydberg states [8,9]. In the following sections, we discuss the assignments of the photoabsorption peaks and photochemical properties of the excited states on the basis of the measurements of the excitation spectra and polarization anisotropy.

\subsection{1. $2 \pi \rightarrow n s \sigma$ Rydberg transition}

The absorption bands observed in the 71000 $78000 \mathrm{~cm}^{-1}$ region have been assigned to the leading members of the $2 \pi \rightarrow n \mathrm{~s} \sigma$ Rydberg series by Felps and co-workers [5-7]. The vibrational structures are characteristic of a linear-linear transition of triatomic molecule, i.e. the vibrational 


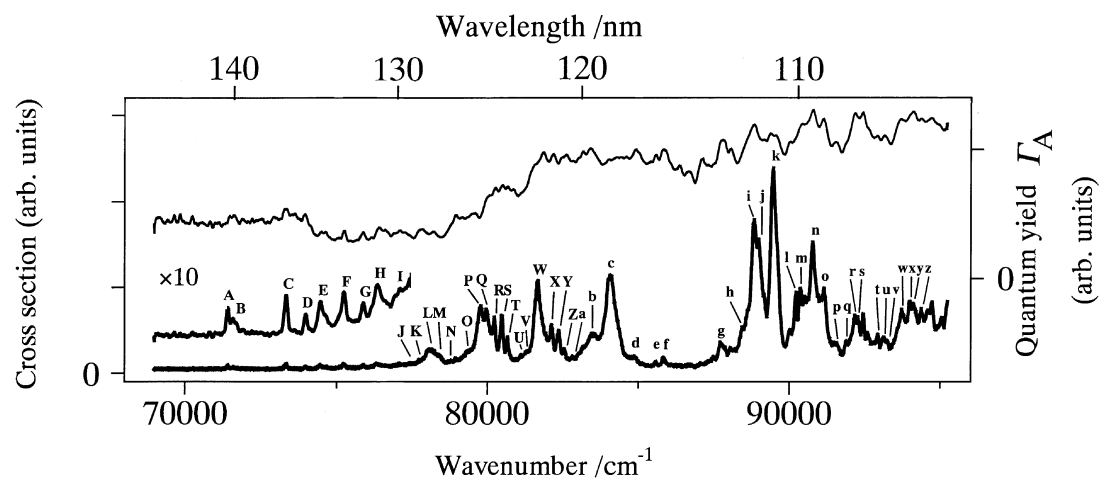

Fig. 3. Relative cross-section (-) and quantum yield (-) for the production of $\mathrm{CN}\left(\mathrm{A}^{2} \Pi_{i}\right)$ from $\mathrm{ClCN}$ in the $69000-94000 \mathrm{~cm}^{-1}$ range.

band associated with the $v_{2}$ mode (bending vibration) was not observed except for very weak 71705 $\mathrm{cm}^{-1}$ band. The fragment $\mathrm{CN}(\mathrm{B}-\mathrm{X})$ emission intrinsically processes negative polarization because a sharp dip is observed at the center of the intense absorption peaks in this region of the $R-\tilde{v}$ curve. This indicates that the photoexcitation at the band center results in a negative $R$ value of the $\mathrm{CN}(\mathrm{B}-$ $\mathrm{X})$ emission. The dependence of polarization on $\tilde{v}$ is characteristic of the $\mathrm{Q}$ branch excitation of a perpendicular-type transition of $\mathrm{ClCN}$ [18]. The quantum defect for band origin at $71398 \mathrm{~cm}^{-1}$ with respect to the first ionization potential is calculated to be 1.03 .

The baseline of the excitation spectra is not zero, which is ascribable to the contribution of an underlying continuum. The $R$ value takes $\approx 0.09$ in the excitation energy region, where the underlying continuum makes a dominant contribution to the production of $\mathrm{CN}(\mathrm{B})$. The positive $R$ value indicates that the photoexcitation responsible for the $\mathrm{CN}(\mathrm{B})$ production occurs through a parallel-type transition of $\mathrm{ClCN}$ [18]. This underlying continuum is assignable to the high-energy tail of the $\alpha$ continuum (the $2 \pi \rightarrow 3 \pi$ intravalence excitation), which extends over $62000-70600 \mathrm{~cm}^{-1}$ [7]. The large magnitude of $R$, which is close to the limiting value, 0.1 , is also consistent with the operation occurrence of direct photodissociation via the $\alpha$ continuum. A non-zero $\Gamma_{\mathrm{B}}$ above $71000 \mathrm{~cm}^{-1}$ obviously conflicts with the semiquantitative prediction based on a simple one-electron MO model that the threshold energy for the $\mathrm{Cl}+\mathrm{CN}(\mathrm{B})$ channel is predicted to locate at $110140 \mathrm{~cm}^{-1}$ [7]. The vibrational analysis of this band system is listed in Table 2.

The peak at $85822 \mathrm{~cm}^{-1}$ (f) is accompanied with $v_{3}$ and $v_{1}+v_{3}$ vibrational bands at $87750(\mathrm{~g})$ and $88449 \mathrm{~cm}^{-1}$ (h). These structures of vibrational progression resemble those of peaks (A), (C) and (D). Dips in the $R-\tilde{v}$ curve show that perpendicular-type transition is responsible for this band system. Therefore, this band system is assignable to the $2 \pi \rightarrow 4 s \sigma\left({ }^{1} \Pi\right)$ Rydberg transition. The band origin of the $2 \pi \rightarrow 5 \mathrm{~s} \sigma\left({ }^{1} \Pi\right)$ Rydberg transition is assigned to the peak at $92268 \mathrm{~cm}^{-1}$ (s).

\subsection{2. $2 \pi \rightarrow 6 \sigma$ intravalence transition}

In the energy range $74000-77000 \mathrm{~cm}^{-1}$, another vibrational progressions (E, H and I) appear in the photoexcitation spectrum of $\mathrm{ClCN}$. Because these bandwidths are clearly wider than near Rydberg bands i.e. $2 \pi \rightarrow 3 \mathrm{~s} \sigma$, the dissociation dynamics through these electronic states should be different from the Rydberg states. Felps et al. have assigned these peaks to the $2 \pi \rightarrow 6 \sigma\left({ }^{1} \Pi\right)$ intravalence transition [7]. These peaks are considered to arise from a perpendicular-type transition because the $R-\tilde{v}$ curve shows a minimum at the center of these peaks. Our experimental result reinforces their assignments. The present vibrational analysis of this band system is shown in Table 3 . 
Table 2

Wave numbers/wavelengths and assignments of Rydberg transitions in the vacuum UV absorption spectrum of ClCN

\begin{tabular}{|c|c|c|c|c|c|c|}
\hline Peak $^{\mathrm{a}}$ & $\tilde{v}\left(\mathrm{~cm}^{-1}\right)^{\mathrm{b}}$ & $\lambda(\mathrm{nm})^{\mathrm{c}}$ & $\Delta \tilde{v}\left(\mathrm{~cm}^{-1}\right)^{\mathrm{d}}$ & $n$ & Assignment & $\delta^{\mathrm{e}}$ \\
\hline \multicolumn{7}{|c|}{$2 \pi \rightarrow n \mathrm{~s} \sigma\left({ }^{1} \Pi\right)$} \\
\hline A & 71398 & 140.06 & & 3 & $0_{0}^{0}$ & 1.03 \\
\hline B & 71705 & 139.46 & 307 & & $2^{1}$ & \\
\hline $\mathrm{C}$ & 73346 & 136.38 & 1948 & & $3^{1}$ & \\
\hline $\mathrm{D}$ & 73975 & 135.18 & 629 & & $1^{1} 3^{1}$ & \\
\hline $\mathrm{F}$ & 75233 & 132.94 & 1887 & & $3^{2}$ & \\
\hline G & 75884 & 131.78 & 651 & & $1^{1} 3^{2}$ & \\
\hline $\mathrm{f}$ & 85822 & 116.52 & & 4 & $0_{0}^{0}$ & 1.20 \\
\hline g & 87750 & 113.96 & 1928 & & $3^{1}$ & \\
\hline $\mathrm{h}$ & 88449 & 113.06 & 699 & & $1^{1} 3^{1}$ & \\
\hline $\mathrm{m}$ & 90367 & 110.66 & 1918 & & $1^{1} 3^{2}$ & \\
\hline s & 92268 & 108.38 & & 5 & $0_{0}^{0}$ & 1.18 \\
\hline $\mathrm{t}$ & 92937 & 107.60 & 669 & & $1^{1}$ & \\
\hline $\mathrm{y}$ & 94091 & 106.28 & 1823 & & $3^{1}$ & \\
\hline \multicolumn{7}{|c|}{$2 \pi \rightarrow n \mathrm{p} \pi\left({ }^{1} \Sigma^{+}\right)$} \\
\hline $\mathrm{P}$ & 79745 & 125.40 & & 3 & $0_{0}^{0}$ & 0.66 \\
\hline $\mathrm{W}$ & 81673 & 122.44 & 1928 & & $3^{1}$ & \\
\hline $\mathrm{b}$ & 83556 & 119.68 & 1883 & & $3^{2}$ & \\
\hline $\mathrm{j}$ & 88984 & 112.38 & & 4 & $0_{0}^{0}$ & (triplet) \\
\hline $\mathrm{k}$ & 89445 & 111.80 & & 4 & $0_{0}^{0}$ & 0.74 \\
\hline 1 & 90204 & 110.86 & 759 & & $1^{1}$ & \\
\hline $\mathrm{p}$ & 91458 & 109.34 & 2013 & & $3^{1}$ & \\
\hline $\mathrm{r}$ & 92166 & 108.50 & 708 & & $1^{1} 3^{1}$ & \\
\hline $\mathrm{x}$ & 93985 & 106.40 & 1819 & & $1^{1} 3^{2}$ & \\
\hline $\mathrm{w}$ & 93738 & 106.68 & & 5 & $0_{0}^{0}$ & 0.74 \\
\hline $\mathrm{z}$ & 94357 & 106.28 & 619 & & $1^{1}$ & \\
\hline \multicolumn{7}{|c|}{$2 \pi \rightarrow n \mathrm{~d} \sigma\left({ }^{1} \Pi\right)^{\mathrm{f}}$} \\
\hline o & 91125 & 109.74 & & 4 & $0_{0}^{0}$ & 0.44 \\
\hline $\mathrm{q}$ & 91878 & 108.84 & 753 & & $1^{0}$ & \\
\hline $\mathrm{u}$ & 93127 & 107.38 & 2002 & & $3^{1}$ & \\
\hline \multicolumn{7}{|c|}{$4 \sigma \rightarrow n s \sigma\left({ }^{1} \Sigma^{+}\right)$} \\
\hline $\mathrm{c}$ & 84076 & 118.94 & & 3 & $0_{0}^{0}$ & 0.99 \\
\hline $\mathrm{d}$ & 84846 & 117.86 & 770 & & $1^{0}$ & \\
\hline e & 85587 & 116.84 & 741 & & $1^{2}$ & \\
\hline \multicolumn{7}{|c|}{$4 \sigma \rightarrow n \mathrm{p} \sigma\left({ }^{1} \Sigma^{+}\right)$} \\
\hline $\mathrm{i}$ & 88842 & 112.56 & & 3 & $0_{0}^{0}$ & 0.79 \\
\hline $\mathrm{n}$ & 90761 & 110.18 & 1919 & & $3^{1}$ & \\
\hline
\end{tabular}

${ }^{a}$ The symbols correspond to those in Figs. 2 and 3.

${ }^{\mathrm{b}}$ Errors in $\tilde{v}$ are estimated to be $\pm 25 \mathrm{~cm}^{-1}$.

${ }^{\mathrm{c}}$ Errors in $\lambda$ are estimated to be $\pm 0.03 \mathrm{~nm}$.

${ }^{\mathrm{d}}$ Frequency interval for the vibrational progressions. These values are comparable to the wave numbers of the corresponding $v_{1}, v_{2}$ and $v_{3}$ modes of $\mathrm{ClCN}^{+}$(see Table 1).

${ }^{\mathrm{e}}$ Quantum defect.

\subsection{3. $2 \pi \rightarrow 4 \pi$ intravalence transition}

The band structures observed at $\approx 78000 \mathrm{~cm}^{-1}$ in the photoexcitation spectrum are too diffuse to identify their band origins and vibrational progressions. Macpherson and Simons have assigned these bands to the ${ }^{3} \Pi$ and ${ }^{1} \Pi$ manifolds arising from the $2 \pi \rightarrow 3 p \sigma$ Rydberg transition [3]. Felps et al. have mentioned this band system only due to Rydberg transitions [7]. The positive $R, \approx 0.09$, which is close to the theoretical limit for direct 
Table 3

Wave numbers/wavelengths and assignments of the intravalence transitions in the vacuum UV absorption spectrum of $\mathrm{ClCN}^{\mathrm{a}}$

\begin{tabular}{lcccl}
\hline Peak & $\tilde{v}\left(\mathrm{~cm}^{-1}\right)$ & $\lambda(\mathrm{nm})$ & $\begin{array}{l}\Delta \tilde{v} \\
\left(\mathrm{~cm}^{-1}\right)\end{array}$ & Assignment \\
\hline $2 \pi \rightarrow$ & & & & \\
E & 74471 & 134.30 & & $0_{0}^{0}$ \\
$\mathrm{H}$ & 76301 & 131.06 & 1830 & $3^{1}$ \\
$\mathrm{I}$ & 76959 & 129.94 & 658 & $1^{1} 3^{1}$ \\
& & & & \\
$2 \pi \rightarrow 4 \pi\left({ }^{1} \Sigma^{+}\right)^{\mathrm{b}}$ & & & \\
$\mathrm{J}$ & 77459 & 129.10 & & $0_{0}^{0}$ \\
$\mathrm{~K}$ & 77761 & 128.60 & 302 & $1^{1}$ \\
$\mathrm{~L}$ & 78064 & 128.10 & 303 & $1^{2}$ \\
$\mathrm{M}$ & 78395 & 127.56 & 331 & $1^{3}$ \\
$\mathrm{~N}$ & 78715 & 127.04 & 320 & $1^{4}$ \\
$\mathrm{O}$ & 79340 & 126.04 & 625 & $1^{6}$ \\
$\mathrm{Q}$ & 79962 & 125.06 & 622 & $1^{8}$ \\
$1 \pi \rightarrow 5 \sigma\left({ }^{1} \Pi\right)$ & & & \\
$\mathrm{R}$ & 80218 & 124.66 & & $0_{0}^{0}$ \\
$\mathrm{~S}$ & 80476 & 124.26 & 258 & $2^{1}$ \\
$\mathrm{~T}$ & 80658 & 123.98 & 182 & $2^{2}$ \\
$\mathrm{U}$ & 81116 & 123.28 & 640 & $1^{1} 2^{1}$ \\
$\mathrm{~V}$ & 81288 & 123.02 & 172 & $1^{1} 2^{2}$ \\
$\mathrm{X}$ & 82129 & 121.76 & 1911 & $3^{1}$ \\
$\mathrm{Y}$ & 82359 & 121.42 & 230 & $2^{1} 3^{1}$ \\
$\mathrm{Z}$ & 82549 & 121.14 & 190 & $2^{2} 3^{1}$ \\
$\mathrm{a}$ & 82823 & 120.74 & 694 & $1^{1} 3^{1}$ \\
\hline
\end{tabular}

${ }^{a}$ See legend of Table 2 .

${ }^{\mathrm{b}}$ Tentative assignments (see text).

dissociation values, indicates that a parallel-type transition makes a dominant contribution to these band structures. It is concluded from this observation that the relevant upper state is dissociative and that its lifetime is much shorter than the rotational period of $\mathrm{ClCN}$. This band system is tentatively assigned to the $2 \pi \rightarrow 4 \pi\left({ }^{1} \Sigma^{+}\right)$intravalence transition, which was also observed in the photoabsorption spectrum of ICN as discussed in Ref. [9]. The vibrational analysis of this band system is listed in Table 3.

\subsection{4. $2 \pi \rightarrow n p \pi$ Rydberg transition}

The most intense absorption band peaking at $79745 \mathrm{~cm}^{-1}$ has been assigned to the $2 \pi \rightarrow 3 \mathrm{p} \pi$ Rydberg transition by Macpherson and Simons [3]. As shown in Fig. 2(b), the $R-\tilde{v}$ curve shows a maximum at the center of the intense absorption peak located at $79745 \mathrm{~cm}^{-1}(\mathrm{P})$. This implies that a parallel-type transition is responsible for this peak. The $R-\tilde{v}$ curve also shows humps at 81673 (W) and $83556(\mathrm{~b}) \mathrm{cm}^{-1}$ peaks, which are assignable to the vibrational members of the $v_{3}$ progressions. Based on these findings, this band system is associated with the $2 \pi \rightarrow 3 \mathrm{p} \pi\left({ }^{1} \Sigma^{+}\right)$transition. Our present result supports the assignment by Macpherson and Simons [3]. The $n=4$ and $n=5$ members of this transition are observed at 89445 $\mathrm{cm}^{-1}(\mathrm{k})$ and $93738 \mathrm{~cm}^{-1}(\mathrm{w})$, respectively, as listed in Table 2.

\subsection{5. $1 \pi \rightarrow 5 \sigma$ intravalence transition}

In the energy range $80200-81300 \mathrm{~cm}^{-1}$, several peaks appear in the photoabsorption spectrum of ClCN. Macpherson et al. and Felps et al. have assigned these peaks to the $4 \sigma \rightarrow 3 \mathrm{~s} \sigma$ and $2 \pi \rightarrow 3 p \pi$ Rydberg transitions, respectively $[3,7]$. However, dips at the center of the absorption peaks are observed in the $R-\tilde{v}$ curve. The dips are also observed at $\approx 82100 \mathrm{~cm}^{-1}$, corresponding to the $v_{3}$ excitation. Felps et al. have estimated the transition energy of the $1 \pi \rightarrow 5 \sigma$ intravalence transition to be $\approx 80460 \mathrm{~cm}^{-1}$ [7]. Based on these observations, this band system is assigned to the $1 \pi \rightarrow 5 \sigma\left({ }^{1} \Pi\right)$ intravalence transition. The molecular structure of this excited state is presumably bent, because the $v_{2}$ (bending vibration) mode progressions were observed. The molecular bond angle could be changed from $180^{\circ}$ by the strong Renner-Teller effect due to the ${ }^{1} \Pi$ type intravalence electronic state. The spectral position of band origin is not obvious. The tentative vibrational analysis of this band system is listed in Table 3 .

\subsection{6. $4 \sigma \rightarrow n s \sigma$ Rydberg transition}

The band at $84076 \mathrm{~cm}^{-1}$ (c) is accompanied with $v_{1}$ and $2 v_{1}$ vibrational bands at 84846 (d) and $85587 \mathrm{~cm}^{-1}$ (e), respectively. The positive $R$ values indicate that parallel-type transition makes a dominant contribution to this band system. The quantum defect for the band origin at $84076 \mathrm{~cm}^{-1}$ with respect to the second ionization potential $\left(113000 \mathrm{~cm}^{-1}\right.$ [14]) is calculated to be 0.99 ; an electron promotion to an s-type Rydberg orbital is involved in the transition [19]. Based on these considerations, this band system is assigned to the $4 \sigma \rightarrow 3 \operatorname{s} \sigma\left({ }^{1} \Sigma^{+}\right)$Rydberg transition (see Table 2). 
This band has the highest quantum yield for the production of $\mathrm{CN}(\mathrm{B})$ in the present observed region. The promotion of the $4 \sigma$ electron preferentially leads to the production of $\mathrm{CN}(\mathrm{B})$, from the molecular orbital correlation between parent $\mathrm{ClCN}$ and $\mathrm{CN}(\mathrm{B})$ fragment.

\subsection{7. $4 \sigma \rightarrow n p \sigma$ Rydberg transition}

The peak located at $88842 \mathrm{~cm}^{-1}$ (i) is assigned to the band origin of the $4 \sigma \rightarrow 3 p \sigma\left({ }^{1} \Sigma^{+}\right)$Rydberg transition because the quantum defect for the origin with respect to the second ionization potential is calculated to be 0.79 , which accords with an electron promotion to a p-type Rydberg orbital [19]. The $R-\tilde{v}$ curve shows a small hump at $90761 \mathrm{~cm}^{-1}$ (n) peak, which is assignable to the vibration member of the $v_{3}$ progression.

\subsection{8. $2 \pi \rightarrow n d \sigma$ Rydberg transition}

The type of absorption transition responsible for the $91125 \mathrm{~cm}^{-1}$ peak (o) cannot be determined definitely from $R$ value, due to the intense neighboring $90761 \mathrm{~cm}^{-1}$ peak (n), which is assigned to the $3^{1}$ band of the $4 \sigma \rightarrow 3 p \sigma$ transition. This band is assignable to the $2 \pi \rightarrow 4 \mathrm{~d} \sigma\left({ }^{1} \Pi\right)$ transition from a quantum defect $\delta \approx 0.44$. The 91878 (q) and $93127 \mathrm{(u} \mathrm{cm}^{-1}$ peaks are assignable to the vibrational members of the $v_{1}$ and $v_{3}$ progression, respectively (see Table 2).

\section{Conclusions}

The high-energy lying states of $\mathrm{ClCN}$ was investigated by the measurement of the excitation spectra and the polarization anisotropy of relevant fluorescence by taking advantage of the linear polarization of synchrotron radiation. Almost dominant absorption bands in the wavelength range 105-145 $\mathrm{nm}$ were assigned on the basis of the symmetry of the upper electronic state. The $2 \pi \rightarrow 4 \pi, 1 \pi \rightarrow 5 \sigma$ intravalence transitions and $4 \sigma \rightarrow n \mathrm{~s} \sigma, 4 \sigma \rightarrow n \mathrm{p} \sigma \quad$ and $2 \pi \rightarrow n \mathrm{~d} \sigma \quad$ Rydberg series were assigned first in the present study. Higher members of the $2 \pi \rightarrow n \mathrm{~s} \sigma, 2 \pi \rightarrow n \mathrm{p} \pi$ Rydberg series could be assigned up. The transitions observed in the photoabsorption spectrum of $\mathrm{ClCN}$ are consistent with those observed in the photoabsorption of $\mathrm{BrCN}$ and ICN, however, the ${ }^{3} \Pi$ manifold was not observed.

Rydberg transitions observed in the present study are converging to the first or second IPs of $\mathrm{ClCN}$. These IPs exhibit in the shorter wavelength region than the present measurement range. We have measured the photoexcited spectra of cyanogen halides in the $30-105 \mathrm{~nm}$ by using the gasfilter apparatus. We will report the assignments and the chemical properties of the high-energy excited states of cyanogen halides in the forthcoming publication.

\section{Acknowledgements}

This work was supported by the Joint Studies Program of the Institute for Molecular Science. K.K. thanks Professor S. Matsui and Dr. Y. Haruyama for their interest and encouragement in the present study.

\section{References}

[1] G.W. King, A.W. Richardson, J. Mol. Spectrosc. 21 (1966) 339.

[2] G.W. King, A.W. Richardson, J. Mol. Spectrosc. 21 (1966) 353.

[3] M.T. Macpherson, J.P. Simons, J. Chem. Soc. Faraday Trans. II 75 (1979) 1572.

[4] M.N.R. Ashfold, A.S. Georgiou, A.M. Quinton, J.P. Simons, J. Chem. Soc. Faraday Trans. II 77 (1981) 259.

[5] W.S. Felps, S.P. McGlynn, G.L. Findley, J. Mol. Spectrosc. 86 (1981) 71.

[6] S.P. McGlynn, W.S. Felps, G.L. Findley, Chem. Phys. Lett. 78 (1981) 89.

[7] W.S. Felps, K. Rupnik, S.P. McGlynn, J. Phys. Chem. 95 (1991) 639.

[8] K. Kanda, S. Katsumata, T. Nagata, Y. Ozaki, T. Kondow, K. Kuchitsu, A. Hiraya, K. Shobatake, Chem. Phys. 175 (1993) 399.

[9] K. Kanda, S. Katsumata, T. Nagata, T. Kondow, A. Hiraya, K. Tabayashi, K. Shobatake, Chem. Phys. 218 (1997) 199.

[10] J.A. Guest, M.A. O’Halloran, R.N. Zare, Chem. Phys. Lett. 103 (1984) 261.

[11] M. Kono, K. Shobatake, J. Chem. Phys. 102 (1995) 5966.

[12] W.C. Fernelius, Inorganic Syntheses, vol. 2, McGraw-Hill, New York, 1946.

[13] L.C. Lee, Chem. Phys. 72 (1980) 6414. 
[14] E. Heilbronner, V. Hornung, K.A. Muszkat, Helv. Chim. Acta 53 (1970) 347.

[15] R.F. Lake, H. Thompson, Proc. Roy. Soc. A 317 (1970) 187.

[16] J.M. Hollas, T.A. Sutherley, Mol. Phys. 22 (1971) 213.

[17] D.H. Whiffen, Spectrochim. Acta 34A (1978) 1173.
[18] M.T. Macpherson, J.P. Simons, R.N. Zare, Mol. Phys. 38 (1979) 2049.

[19] A.B.F. Duncan, Rydberg Series in Atoms and Molecules, Academic Press, New York, 1971.

[20] F.G. Celii, J. Fulara, J.P. Maier, M. Rösslein, Chem. Phys. Lett. 131 (1986) 325. 